\title{
Solving Multi-Objective Linear Fractional Stochastic Transportation Problems Involving Normal Distribution using Simulation-Based Genetic Algorithm
}

\author{
Adane Abebaw Gessesse, Rajashree Mishra, Mitali Madhumita Acharya
}

\begin{abstract}
In real-life situations, we human beings faced with multi-objective problems that are conflicting and non-commensurable with each other. Especially, when goods are transported from source to locations with a goal to keep exact relationships between a few parameters, those parameters of such problems might also arise in the form of fractions which are linear in nature such as; actual transportation fee/total transportation cost, delivery fee/desired path, total return/total investment, etc. Due to the uncertainty of nature, such a relationship is not deterministic. Mathematically such kinds of mathematical problems are characterized as a multi-objective linear fractional stochastic transportation problem. However, it is difficult to handle such types of mathematical problems. It can't be solved directly using mathematical programming approaches. In this paper, a solution procedure is proposed for the above problem using a stochastic Genetic Algorithm based simulation. The parameters in the constraint of the above problem follow a normal distribution. The probabilistic constraints are handled by stochastic simulation-based GA for the solution procedure of the proposed problem. The feasibility of probability constraints is checked by the stochastic programming through the Genetic Algorithm approach, without finding the equivalent deterministic model. The feasibility is maintained all-over the problem. The stochastic simulation-based Genetic Algorithm is considered to generate non-dominated solutions for the given problem. Then, a numerical case study is provided to illustrate the method.
\end{abstract}

Keywords: Genetic Algorithm, multi-objective programming, stochastic fractional programming, transportation problem.

\section{INTRODUCTION}

Transportation problems with the ratio of optimization of parameters where the ratios are objective functions are known as fractional transportation problems. It is concerned with delivering the commodities from numerous assets to various locations along to keep up great connections among a

Revised Manuscript Received on December 30, 2019.

* Correspondence Author

Adane Abebaw Gessesse*, Department of Mathematics, KIIT, Deemed to be University, Bhubneswar 751024, India.

Rajashree Mishra, Department of Mathematics, KIIT, Deemed to beUniversity, Bhubneswar 751024, India.

Mitali Madhumita Acharya, Department of Mathematics, KIIT,Deemed to be University, Bhubaneswar 751024, India.

(C) The Authors. Published by Blue Eyes Intelligence Engineering and Sciences Publication (BEIESP). This is an open access article under the CC BY-NC-ND license (http://creativecommons.org/licenses/by-nc-nd/4.0/) couple of parameters. Those parameters of transportation problems may happen as a proportion of actual transportation cost/total standard transportation cost, shipping cost/desired path, total return/total investment, and so forth.

In real-life, distributions of commodities are done on the minimization of the ratio of the total cost to total profit. The problem derived by such type of two linear functions gets its name as a linear fractional transportation problem (LFTP). In many real-world situations, for LFTP, decisions are often made in the presence of multiple, non-commensurable, conflicting objectives. Such kinds of problems are called multi-objective linear fractional transportation problems (MOLFTP). It deals with the distribution of goods at a time by considering the ratio of several objective functions. The parameters associated with the MOLFTP are not deterministic or fixed value always. In a mathematical programming model, uncertainties are addressed using the fuzzy program set theory or probability theory. In the present paper, we deal with the parameters to address uncertainty using probability theory. The presence of probability in a mathematical programming problem leads to a stochastic programming (SP) problem.

SP problem is one of the mathematical programming problems that involve randomness. It is concerned with the decision-making in which a few or all parameters traced as random variables for capturing uncertainty.

In our proposed work, attention has been given to solve a stochastic transportation problem having more than one linear fractional objective function. The parameters of the constraints in the above problem are normal random variables. The mathematical model is known as a multi-objective linear fractional stochastic transportation problem (MOLFSTP). However, a set of optimal solutions known as Pareto-optimal (PO) solutions occurs due to the presence of conflicting objectives in a MOLFSTP. Finding these set of PO solutions is not practically possible, rather an approximation set to the true Pareto front (PF) is expected. Researchers have attempted various methods to tackle those types of MOLFSTP problems. Nowadays, due to the popularization of the evolutionary algorithm, many researches are going on solving the above problem using the said algorithm. One such popular algorithm is the Genetic Algorithm (GA) which is an efficient algorithm for tackling such type of problems. 


\section{Solving MOLFSTP Involving Normal Distribution Using Simulation Based GA}

Because of its population-based nature, in a single simulation run, GA can obtain multiple PO solutions. GA is superior in comparison to the classical methods. Because it finds convergent solutions, finds a diversified set of solutions, and covers the entire PF [1].

The remainder of the paper is set up as follows. Following the introduction section, the literature survey has been provided in Section 2.

Basic preliminaries are presented in Section 3. The mathematical model is defined and described in Section 4. Simulation-based GA and its solution procedure are presented in Section 5. Case study and results and discussion have been presented in Section 6 and 7, respectively. The concluding remarks are given in Section 8, followed by references.

\section{LITERATURE SURVEY}

Swarup [2] was the first who proposed an LFTP. The systematic development of LFTP is found in [3, 4, 5,6,7].

An algorithm is presented by Gupta and Arora [8] to obtain the best cost-time trade-off pairs in a fractional capacitated transportation problem with bounds upon availabilities and demands. Guzel et al. [9] developed a solution procedure for fractional transportation problems with the interval coefficient. Pradhan and Biswal [10] presented a couple of algorithms to obtain an initial fundamental feasible solution of a linear fractional transportation problem.

In real-world applications, there are cases that the parameters might be inexact and have to be estimated. Due to the lack of exact data, some uncertain factors might occur within the problems. To deal with such a phenomenon,

Liu [11] found the uncertainty theory and redefined it. It was applied to address uncertain problems by many researchers to date $[12,13]$.

For handling uncertainty, different researchers have discussed on SP problem. Dantzig [14] was the first who formulated the SP model. Several researchers have recommended different models on SP $[15,16]$. For handling uncertainty, several researchers have discussed on SP problem.

Many researchers have been developed for stochastic fractional transportation problems and their solution techniques. Charles and Dutta [17] proposed an interactive conversion technique that converts the sum of probabilistic fractional objective into the stochastic constraint with the help of a deterministic parameter. Charles and Dutta [18] applied multi-objective stochastic fractional programming problems to compiled published circuit board problems. Jain and Arya [19] presented an inverse optimization model for the transportation problem of optimizing the ratio of linear functions and linear constraints. Jadhav and Doke [20] presented a solution method to solve the fractional transportation problem wherein the coefficient of the objective function is fuzzy. Javaid, Jalil, and Asim [21] introduced a transportation problem model with a couple of fractional objectives involving random parameters.

Holland [22] developed GA, which is primarily in light of the idea of the biological process of natural selection. Holland and his understudies have devoted a great deal to the advancement of the area.

Many researchers have studied evolutionary computing and its application for solving transportation problems. GA is a well known and effective strategy for such sort of issues. Vignaux and Michalewicz [23] discussed how to solve linear transportation problem using alternative GA. Syarif [24] developed a GA approach for solving nonlinear side constrained transportation problems. Bharathi and Vijayalakshmi [25] presented an application of evolutionary algorithms to the multi-objective transportation problem (MOTP). A solution procedure is presented for a MOTP by a fuzzy stochastic simulation-based GA by Dutta, Acharya and Mishra. [26]. A GA is applied for shipping, location, and allocation of dangerous substances to a novel bi-objective stochastic model [27]. Recently, Karthy and Ganesan [28] applied a GA for solving the MOTP. Going through the literature survey, we were motivated by a work done on MOTP using GA by Dutta, Acharya and Mishra. [26]. Their paper concentrated on fuzzy stochastic simulation-based GA as a solution procedure for a MOTP. The amount and request parameters of the restrictions follow fuzzy-exponential and fuzzy-normal distribution, respectively. However, in our proposed paper, a novel strategy has been evolved for MOLFSTP involving Normal distribution. For solving the proposed model, we implement a simulation-based GA. The main difference between this paper and the above paper is listed as follows. Firstly, this paper concentrated on a ratio of two objective functions. Secondly, both supply and demand parameters are normal random variables. Finally, there are no fuzzy parameters in this paper.

\section{BASIC PRELIMINARIES}

\section{A. Bounded Random Number (BRN)}

The function $\mathrm{rand}(\mathrm{)}$ is one way to generate random numbers between 0 and $R A N D \_M A X$, where $R A N D \_M A X$ is defined in \#stdlib as $\left(2^{10}-1\right)$ in $\mathrm{C}++$. Hence, to generate a random number in $[0,1]$, the following steps are followed.

$$
\begin{aligned}
& * \quad m=\text { rand }() \\
& \text { * } m \leftarrow m / R A N D \_M A X
\end{aligned}
$$

\section{B. Normal Distribution}

It is one of the probability distribution. The parameters which define Normal distribution are mean (location parameter) $\mu$ and standard deviation (scale parameter) $\sigma$.

The probability density function (pdf) of Normal distribution is defined as:

$$
f(x)=\frac{1}{\sqrt{2 \pi} \sigma} \exp \left\{-\frac{(x-\mu)^{2}}{2 \sigma^{2}}\right\}
$$

where $x>0, \mu$ is mean, $\sigma>0$ is standard deviation.

For generating Normal distribution, the following steps are used.

Step-1: Generate $m$ and $n$ from $B R N(0,1)$.

Step-2: Use mean $\mu$ and standard deviation $\sigma$.

Step-3: Return $z=[-2 \ln (n)]^{1 / 2} \sin (2 \pi n)$.

Step-4: Return $\mu+\sigma z$

\section{Stochastic Simulation for Probabilistic Constraints}

In a stochastic condition, some or all the coefficients of a probabilistic constraint may be random variables with a known probability distribution. For the probabilistic constraints where randomness occurs on the right-hand side are defined in (2) and (3). 


$$
\begin{gathered}
P\left(\sum_{t=1}^{n} x_{s t} \leq a_{s}\right) \geq 1-\gamma_{s} ; s=1,2, \ldots, m \\
P\left(\sum_{s=1}^{m} x_{s t} \geq b_{t}\right) \geq 1-\delta_{t} ; t=1,2, \ldots, n
\end{gathered}
$$

Let's define $U_{s}\left(r_{1}, x\right)$ and $W_{t}\left(r_{2}, x\right)$ as follows:

$$
\begin{aligned}
& U_{s}\left(r_{1}, x\right)=\sum_{t=1}^{n} x_{s t}-a_{s} ; s=1,2, \ldots, m \\
& W_{t}\left(r_{2}, x\right)=\sum_{s=1}^{m} x_{s t}-b_{t} ; t=1,2, \ldots, n
\end{aligned}
$$

The probability constraints as defined in (2) and (3) can be written as follows:

$$
P\left(U_{s}\left(r_{1}, x\right) \leq 0\right) \geq 1-\gamma_{s} ; s=1,2, \ldots, m
$$

$$
P\left(W_{t}\left(r_{2}, x\right) \geq 0\right) \geq 1-\delta_{t} ; t=1,2, \ldots, n
$$

Where $r_{1}=\left(a_{1}, a_{2}, \ldots, a_{m}\right)$ and $r_{2}=\left(b_{1}, b_{2}, \ldots, b_{n}\right)$ are an $m$-dimensional and $n$-dimensional vector of random numbers respectively, $x=\left(x_{1 t}, x_{2 t}, \ldots, x_{m t}\right) ; t=1,2, \ldots, n$ is the vector of decision variables and $\gamma_{s} ; s=1,2, \ldots, m$ and $\delta_{t} ; t=1,2, \ldots, n$ are pre specified confidence levels.

$N$ independent random vectors $r_{1}^{i}=\left(a_{1}^{i}, a_{2}^{i}, \ldots, a_{m}^{i}\right) ; i=$ $1,2, \ldots, N$ and $M$ independent random vectors $r_{2}^{j}=$ $\left(b_{1}^{j}, b_{2}^{j}, \ldots, b_{n}^{j}\right) ; j=1,2, \ldots, M$ are generated where $a_{s}^{i}$ and $b_{t}^{j}$ are random numbers generated according to the distribution of $a_{s}$ and $b_{t}$ respectively. Let $N_{s}^{\prime}(s=1,2 \ldots, m)$ and $M_{t}^{\prime}(t=1,2, \ldots, n$ be the number of cases in which $U_{s}\left(r_{1}, x\right) \leq 0 ; s=1,2, \ldots, m$ and $W_{t}\left(r_{2}, x\right) \geq 0 ; t=1,2, \ldots, n$ respectively.

Then by the definition of probability, (2) and (3) hold if $N_{s}^{\prime} /_{N} \geq 1-\gamma_{s}$ and $M_{t}^{\prime} /_{M} \geq 1-\delta_{t}$ respectively for $s=$ $1,2, \ldots, m ; t=1,2, \ldots, n$.

\section{Feasibility of Probability Constraints}

For checking the feasibility of the probability constraints of the right hand side parameters, the following steps are used.

Step-1: Use all the steps for generating Normal distribution. i.e.,

* Generate $r_{s}, r_{s}^{\prime}, r_{t}$ and $r_{t}^{\prime}$ from $B R N(0,1)$.

* Use mean $\mu_{s}, \mu_{t}^{\prime}$ and standard deviation $\sigma_{s}, \sigma_{t}^{\prime}$.

* Return $z_{s}=\left[-2 \ln \left(r_{s}^{\prime}\right)\right]^{1 / 2} \sin \left(2 \pi r_{s}\right)$ and

$$
z_{t}=\left[-2 \ln \left(r_{t}^{\prime}\right)\right]^{1 / 2} \sin \left(2 \pi r_{t}\right)
$$

* Return $T_{s}=\mu_{s}+\sigma_{s} z_{s}$ and $T_{t}^{\prime}=\mu_{t}^{\prime}+\sigma_{t}^{\prime} z_{t}$

Step-2: Return $T=\sum_{s=1}^{m} T_{s}$ and $T^{\prime}=\sum_{t=1}^{n} T_{t}^{\prime}$

Step-3: Return $P=T-T^{\prime}$

Step-4: If $P \geq 0$, then the generated population is feasible for the probability constraints.

\section{MATHEMATICAL MODEL OF MOLFSTP}

Mathematically, a MOLFSTP where randomness is considered in the right-hand-side constraints is expressed as:

$$
\operatorname{Min}: Z^{l}=\frac{\sum_{\mathrm{s}=1}^{\mathrm{m}} \sum_{\mathrm{t}=1}^{\mathrm{n}} c_{\mathrm{st}}^{\mathrm{l}}}{\sum_{\mathrm{s}=1}^{\mathrm{m}} \sum_{\mathrm{t}=1}^{\mathrm{n}} p_{\mathrm{st}}^{\mathrm{l}}} ; l=1,2, \ldots, L
$$

Subject to:

$$
\begin{aligned}
& P\left(\sum_{t=1}^{n} x_{s t} \leq a_{s}\right) \geq 1-\gamma_{s} ; s=1,2, \ldots, m \\
& P\left(\sum_{s=1}^{m} x_{s t} \geq b_{t}\right) \geq 1-\delta_{t} ; t=1,2, \ldots, n
\end{aligned}
$$

where $x_{s t} \geq 0,0<\gamma_{s}, \delta_{t}<1 ; \forall s, t$.

Let $a_{s}(s=1,2, \ldots, m)$ and $b_{t}(t=1,2, \ldots, n)$ are normal random variables. The unit shipping cost coefficients along the traveled route and preferring route for transporting of goods from source $s$ to destination $t$ is represented by $c_{s t}^{l}$ and $\quad p_{s t}^{l}(s=1,2, \ldots, m ; t=1,2, \ldots, n ; l=1,2, \ldots, L)$ respectively. $\gamma_{s}$ and $\delta_{t}$ are pre specified probability levels for all $s, t$. The variable $x_{s t}$ denotes the amount transported from source $s$ to destination $t$. It is expected that the denominator of the objective function remains positive, and the total supply is greater than or equal to total demand.

\section{SIMULATION BASED GA FOR MOLFSTP}

The method is designed to solve the MOLFSTP. The algorithmic steps are described as follows:

Step-1: Fix GA parameters and termination criteria (Max_gen)

Step-2: Generate the parameters for the given distribution.

Step-3: Initialize the GA population for the objective function $Z^{l}(l=1)$ with the given constraints.

Step-4: Initialize generation gen $=1$ and penalty parameter $\tau$

Step-5: Apply the bounds on the population and calculate constraints for each objective function.

Step-6: Check the feasibility condition, if satisfied go to Step 7 else go to Step 3.

Step-7: Probability criteria is checked, if satisfied go to Step 8 else go to Step 3.

Step-8: Calculate the functional value i.e., the objective function.

Step-9: Apply Selection, Crossover and Mutation respectively.

Step-10: Again, calculate the functional value i.e., the objective function.

Step-11: Again check feasibility criteria, if satisfied go to Step 12 else go to Step 3.

Step-12: Again check probability criteria, if satisfied go to Step 13 else go to Step 3.

Step-13: Apply Elitism.

Step-14: Check the stopping criteria. If reached, the current population is the best population else gen $=$ gen + 1 and go to Step 5.

Step-15: Ideal solution is obtained for the first objective function.

Step-16: Repeat the steps from Step 2 to Step 15 for the other objective function until ideal solution obtained for all objective functions. 


\section{Solving MOLFSTP Involving Normal Distribution Using Simulation Based GA}

Step-17: Construct a pay-off matrix containing the ideal solution and functional values as shown in Table I.

Step-18: Formulate the fitness function by using bracket penalty operator:

Table I: Pay-Off Matrix

\begin{tabular}{|c|c|c|c|c|}
\hline \multirow{2}{*}{$\begin{array}{l}\text { Ideal } \\
\text { Solutions }\end{array}$} & \multicolumn{4}{|c|}{ Objective functions } \\
\cline { 2 - 5 } & $Z^{1}(x)$ & $Z^{2}(x)$ & $\cdots$ & $Z^{L}(x)$ \\
\hline$X^{(1)}$ & $Z^{1}\left(X^{(1)}\right)$ & $Z^{2}\left(X^{(1)}\right)$ & $\cdots$ & $Z^{L}\left(X^{(1)}\right)$ \\
\hline$X^{(2)}$ & $Z^{1}\left(X^{(2)}\right)$ & $Z^{2}\left(X^{(2)}\right)$ & $\cdots$ & $Z^{L}\left(X^{(2)}\right)$ \\
\hline$\vdots$ & $\vdots$ & $\vdots$ & $\vdots$ & $\vdots$ \\
\hline$X^{(L)}$ & $Z^{1}\left(X^{(L)}\right)$ & $Z^{2}\left(X^{(L)}\right)$ & $\cdots$ & $Z^{L}\left(X^{(L)}\right)$ \\
\hline
\end{tabular}

$$
F^{l}(x)=Z^{l}(x)+\tau \sum_{i=1}^{s+t}<g^{i}(x)>^{2} ; l=1,2, \ldots, L
$$

where

$$
\begin{gathered}
Z^{l}(x)=\frac{\sum_{\mathrm{s}=1}^{\mathrm{m}} \sum_{\mathrm{t}=1}^{\mathrm{n}} c_{\mathrm{st}}^{\mathrm{l}}}{\sum_{\mathrm{s}=1}^{\mathrm{m}} \sum_{\mathrm{t}=1}^{\mathrm{n}} p_{\mathrm{st}}^{\mathrm{l}}} ; l=1,2, \ldots, L \\
g^{i}(x)=\sum_{t=1}^{n} x_{s t}-\left(\mu_{\mathrm{s}}+\sigma_{s} z_{s}\right) ; i=1,2, \ldots, s ; \\
g^{i}(x)=\sum_{s=1}^{m} x_{s t}-\left(\mu_{t}^{\prime}+\sigma_{t}^{\prime} z_{t}\right) ; \\
\quad i=s+1, s+2, \ldots, s+t .
\end{gathered}
$$

where $x_{s t} \geq 0 ; \forall s, t . F^{l}(x)$ is a fitness function, $Z^{l}(x)$ is objective function, $\tau$ is penalty parameter, $z_{s}$ and $z_{t}$ are as described in Section $3.4,<\mathrm{g}^{i}(x)>$ is constraint violation in which $\langle\cdot\rangle$ denotes the absolute value of the operand where $<g>=\left\{\begin{array}{l}0, \mathrm{~g} \geq 0 \\ \mathrm{~g}, \mathrm{~g}<0\end{array}\right.$

Step-19: Solve using GA to obtain Pareto optimal solutions.

The algorithmic steps described earlier are displayed as a flow diagram in Fig. 1.

\section{CASE STUDY}

GAA-Oil mining"(name changed) company mines from three branches to supply the oil for five cities in India. The manager of the company decided to plan for transportation for the next month onwards. He needs to collect the primary records along with delivery capacity, demand, total profit, cost of a unit product, shipping time, and so on at the start of his project. However, due to uncertain human and natural phenomena, he can't get these data exactly. According to previous experiences, the company assumes that the supply and demand parameters follow a normal uncertain distribution with known mean and standard deviation.

The production cost and profit per unit (in liters) from source to destination are given in Table II. Similarly, the delivery time from the source to the destination for a unit is given in Table III.

Table II: Production cost/profit per unit (in rupees)

\begin{tabular}{|l|l|l|l|l|l|}
\hline \multirow{2}{*}{ Source } & \multicolumn{4}{|l|}{ Destination } \\
\cline { 2 - 6 } & City 1 & City 2 & City 3 & City 4 & City 5 \\
\hline Branch 1 & $18 / 30$ & $17 / 32$ & $18 / 34$ & $18 / 30$ & $20 / 32$ \\
\hline Branch 2 & $10 / 20$ & $10 / 18$ & $12 / 22$ & $9 / 20$ & $10 / 16$ \\
\hline Branch 3 & $20 / 40$ & $18 / 32$ & $20 / 32$ & $22 / 30$ & $18 / 36$ \\
\hline
\end{tabular}

Table III: Delivery time (actual/standard) per unit (in hours)

\begin{tabular}{|l|l|l|l|l|l|}
\hline \multirow{2}{*}{ Source } & \multicolumn{4}{|l|}{ Destination } \\
\cline { 2 - 6 } & City 1 & City 2 & City 3 & City 4 & City 5 \\
\hline Branch 1 & $10 / 12$ & $8 / 10$ & $10 / 13$ & $10 / 12$ & $12 / 15$ \\
\hline Branch 2 & $6 / 8$ & $6 / 9$ & $7 / 10$ & $5 / 7$ & $6 / 9$ \\
\hline Branch 3 & $12 / 14$ & $10 / 13$ & $12 / 15$ & $13 / 18$ & $10 / 12$ \\
\hline
\end{tabular}

The mathematical model for the above problem is expressed as below in (12) to (15).

$\min : Z^{1}(x)=\frac{\sum_{s=1}^{3} \sum_{\mathrm{t}=1}^{5} c_{s t}}{\sum_{\mathrm{s}=1}^{3} \sum_{\mathrm{t}=1}^{5} p_{s t}}$

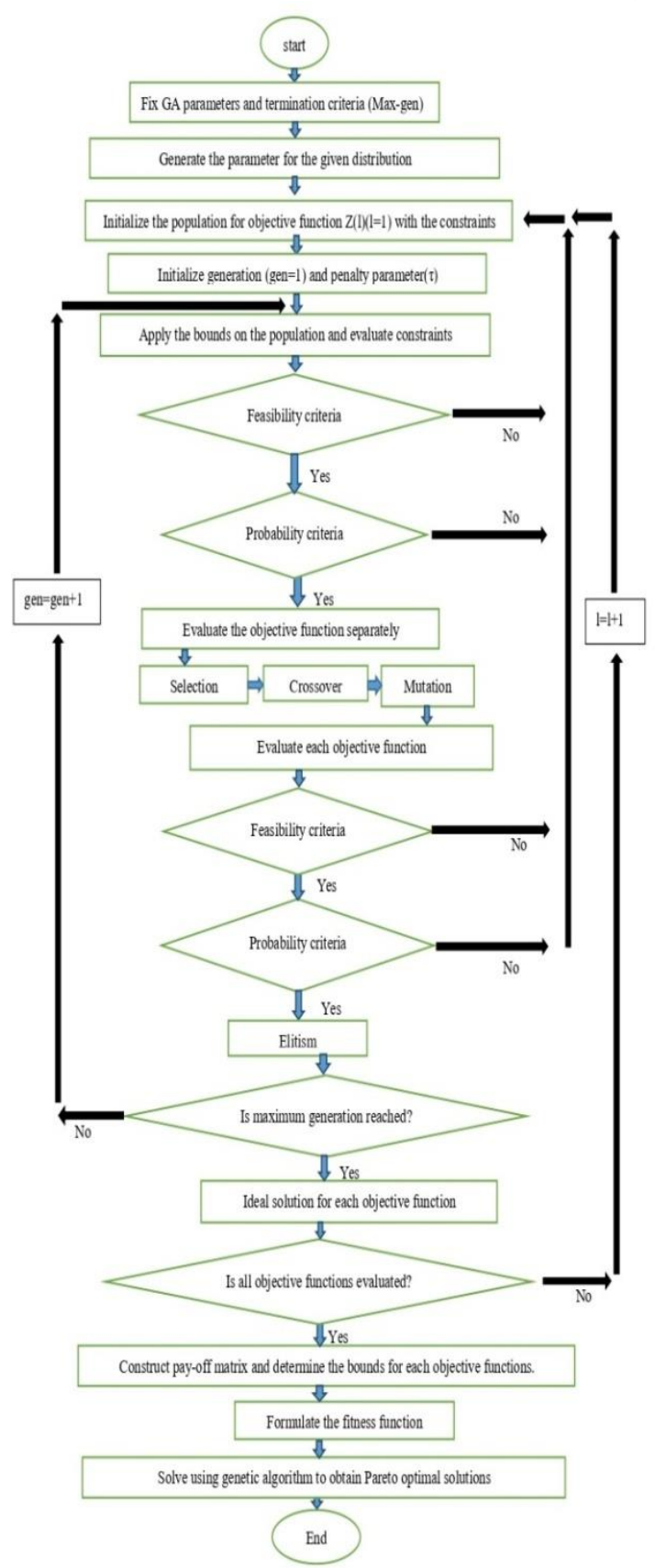

Fig. 1: Flow Diagram of Simulation based GA Approach to solve MOLFSTP 


$$
Z^{2}(x)=\frac{\sum_{s=1}^{3} \sum_{t=1}^{5} A_{s t}}{\sum_{s=1}^{3} \sum_{t=1}^{5} S_{s t}}
$$

Subject to:

$$
\begin{aligned}
& P\left(\sum_{t=1}^{5} x_{s t} \leq a_{s}\right) \geq 1-\gamma_{s} ; s=1,2,3 . \\
& P\left(\sum_{s=1}^{3} x_{s t} \geq b_{t s}\right) \geq 1-\sigma_{t} ; t=1,2,3,4,5 .
\end{aligned}
$$

$x_{s t} \geq 0$ and $0<\gamma_{s}, \delta_{t}<1 ; \forall s, t$.

The known parameters of a normal distribution with a specified probability level (SPL) of supplies $a_{s}(s=1,2,3)$ and demands $b_{t}(1,2,3,4,5)$ are presented in Table IV and Table $\mathrm{V}$ respectively.

Table IV: Values of SPL; mean and standard deviation for supplies $a_{s}$

\begin{tabular}{|c|c|c|}
\hline Mean & standard deviation & SPL $\left(\gamma_{s}\right)$ \\
\hline$\mu_{1}=24$ & $\sigma_{1}=2$ & $\gamma_{1}=0.9$ \\
\hline$\mu_{2}=32$ & $\sigma_{2}=1.5$ & $\gamma_{2}=0.9$ \\
\hline$\mu_{3}=30$ & $\sigma_{3}=2$ & $\gamma_{3}=0.9$ \\
\hline
\end{tabular}

Table V: Values of SPL; mean and standard deviation for supplies $\boldsymbol{b}_{t}$

\begin{tabular}{|c|c|c|}
\hline Mean & standard deviation & SPL $\left(\delta_{t}\right)$ \\
\hline$\mu_{1}^{\prime}=12$ & $\sigma_{1}^{\prime}=1.5$ & $\delta_{1}=0.9$ \\
\hline$\mu_{2}^{\prime}=10$ & $\sigma_{2}^{\prime}=1$ & $\delta_{2}=0.9$ \\
\hline$\mu_{3}^{\prime}=16$ & $\sigma_{3}^{\prime}=1$ & $\delta_{3}=0.9$ \\
\hline$\mu_{4}^{\prime}=10$ & $\sigma_{4}^{\prime}=1.5$ & $\delta_{4}=0.9$ \\
\hline$\mu_{5}^{\prime}=14$ & $\sigma_{5}^{\prime}=1$ & $\delta_{5}=0.9$ \\
\hline
\end{tabular}

Using the data in Tables IV and V, the above multi-objective fractional stochastic transportation problem is solved using simulation-based GA. Applying the steps of simulation based GA, we obtain two ideal solutions as

$$
X^{(1)}=(0.2541,0.3187,0.9834,0.1945,0.8094,0.1222 \text {, }
$$
$0.6989,0.1251,0.5415,0.0117,0.3744,0.1642$, $0.0694,0.4242,0.8113)$

$X^{(2)}=(0.2678,0.7146,0.3646,0.1642,0.0156,0.0938$ $0.2991,0.7928,0.3167,0.1896,0.0557,0.1320$, $0.6188,0.7947,0.2727)$.

The objective function values are $Z^{1}\left(X^{(1)}\right)=0.5554$ and $Z^{2}\left(X^{(2)}\right)=0.7649$ respectively.

Using the two ideal solutions a pay-off matrix is formulated in Table VI.

Table VI: Pay-Off Matrix for case study

\begin{tabular}{|c|c|c|}
\hline \multirow{2}{*}{$\begin{array}{l}\text { Ideal } \\
\text { Solutions }\end{array}$} & \multicolumn{2}{|c|}{ Objective functions } \\
\cline { 2 - 3 }$X^{(1)}$ & 0.5554 & $Z^{1}$ \\
\hline$X^{(2)}$ & 0.5833 & 0.7777 \\
\hline
\end{tabular}

Applying bracket penalty function MOLFSTP is solved as follows in (16) to (20).

$$
\begin{aligned}
& F^{1}(x)=Z^{1}(x)+\tau \sum_{i=1}^{8}<g^{i}(x)>^{2} \\
& F^{2}(x)=Z^{2}(x)+\tau \sum_{i=1}^{8}<g^{i}(x)>^{2}
\end{aligned}
$$

$$
\begin{gathered}
Z^{1}(x)=\frac{\sum_{s=1}^{3} \sum_{\mathrm{t}=1}^{5} c_{s t}}{\sum_{\mathrm{s}=1}^{3} \sum_{\mathrm{t}=1}^{5} p_{s t}} \\
Z^{2}(x)=\frac{\sum_{\mathrm{s}=1}^{3} \sum_{\mathrm{t}=1}^{5} A_{s t}}{\sum_{\mathrm{s}=1}^{3} \sum_{\mathrm{t}=1}^{5} S_{s t}} \\
g^{i}(x)=\sum_{t=1}^{5} x_{s t}-\left(\mu_{s}+\sigma_{s} z_{s}\right) ; i=1,2,3 ; \\
g^{i}(x)=\sum_{s=1}^{3} x_{s t}-\left(\mu_{t}^{\prime}+\sigma_{t}^{\prime} z_{t}^{\prime}\right) ; i=4, \ldots, 8 .
\end{gathered}
$$

\section{RESULT AND DISCUSSION}

The proposed simulation based GA approach is coded in C++ Code:: Blocks 16.01 compiler. The size of population is taken as 100 . The numbers of generations are taken to be 100 . The penalty parameter is taken for an initial value of $\tau=10$ and it is incremented by 10 after $10^{\text {th }}$ generation. The ideal solutions are recorded by taking more than 10 simulations. However, the top 4 values are record. An extensive experimental study has been done varying the value of probability of crossover $\left(P_{c}\right)$ and probability of mutation $\left(P_{m}\right)$ respectively. The $P_{c}$ has been taken $0.6,0.7,0.8$, and 0.9 and $P_{m}$ has been taken $0.001,0.005,0.01,0.05$, and 0.1 .

In these simulations, the most approximate redundant values are taken as ideal solution for each objective function. PO solutions and values are obtained and shown in Tables (VIII-XI) for different values of $P_{c}$ and $P_{m}$ respectively.

The best functional values are recurred as shown below for different values of $P_{c}$ and $P_{m}$.

Table VII: Best functional values

\begin{tabular}{|c|c|c|c|c|c|}
\hline \multirow{3}{*}{$P_{m}$} & \multicolumn{5}{|c|}{$P_{c}$} \\
\cline { 2 - 6 } & $\begin{array}{l}\text { Objective } \\
\text { function } \\
\text { value }\end{array}$ & 0.6 & 0.7 & 0.8 & 0.9 \\
\hline 0.001 & $Z^{1}$ & 0.5461 & 0.5508 & 0.5581 & 0.5451 \\
\cline { 2 - 6 } & $Z^{2}$ & 0.7590 & 0.7771 & 0.7716 & 0.7618 \\
\hline 0.005 & $Z^{1}$ & 0.5547 & 0.5576 & 0.5490 & 0.5541 \\
\cline { 2 - 6 } & $Z^{2}$ & 0.7761 & 0.7744 & 0.7610 & 0.7730 \\
\hline 0.01 & $Z^{1}$ & 0.5512 & 0.5552 & 0.5509 & 0.5459 \\
\cline { 2 - 6 } & $Z^{2}$ & 0.7694 & 0.7610 & 0.7718 & 0.7788 \\
\hline 0.05 & $Z^{1}$ & 0.5521 & 0.5517 & 0.5489 & 0.5483 \\
\cline { 2 - 6 } & $Z^{2}$ & 0.7766 & 0.7756 & 0.7662 & 0.7761 \\
\hline 0.1 & $Z^{1}$ & 0.5498 & 0.5557 & 0.5449 & 0.5609 \\
\cline { 2 - 6 } & $Z^{2}$ & 0.7743 & 0.7747 & 0.7763 & 0.7722 \\
\hline
\end{tabular}

From the tabular results, it can be seen that for both of the objectives the best functional values are obtained at $P_{c}=0.6$ and $P_{m}=0.001$, with functional value $Z^{1}=0.5461$ and $Z^{2}=0.7590$. However, at $P_{c}=0.7$, the best functional value for the first objective is $Z^{1}=0.5508$ at $P_{m}=0.001$ and for the second objective is $Z^{2}=0.7610$ at $P_{m}=0.01$. It is also observed, at $\left(P_{c}=0.8\right)$ the best functional value for the first objective is $Z^{1}=0.5449$ at $\left(P_{m}=0.1\right)$ and for the second objective is $Z^{2}=0.7610$ at $\left(P_{m}=0.005\right)$. Lastly, at $\left(P_{c}=0.9\right)$ and 
$\left(P_{m}=0.001\right)$, the best functional values for both of the objectives are $Z^{1}=0.5451$ and $Z^{2}=0.7618$ respectively. Graphical representations of the PO values are expressed in Fig. 2 to Fig. 5. From the plotted figures diversified PO values can be visualized for different values of $P_{c}$ and $P_{m}$.

\section{CONCLUSION}

In this study, a stochastic simulation-based GA is used to solve the proposed MOLFSTP model without deriving the equivalent deterministic model. Stochastic simulation-based GA is superior in comparison to classical methods. It supports the decision-maker in forming a collection of non-dominated solutions, to obtain a diversified solution, and to cover the entire Pareto front. This also helps the decision-maker to make a more favorable choice by analyzing all the desirable way of the parameter. The non-dominated solutions represent the positions in this solution space of the problem. A numerical case study is provided to illustrate the methodology where both the supply and demand points follow a normal distribution. It is concluded from the tabular results shown in Table (VIII-XI) that the diversified Pareto optimal values can be visualized for different values of $P_{c}$ and $P_{m}$.

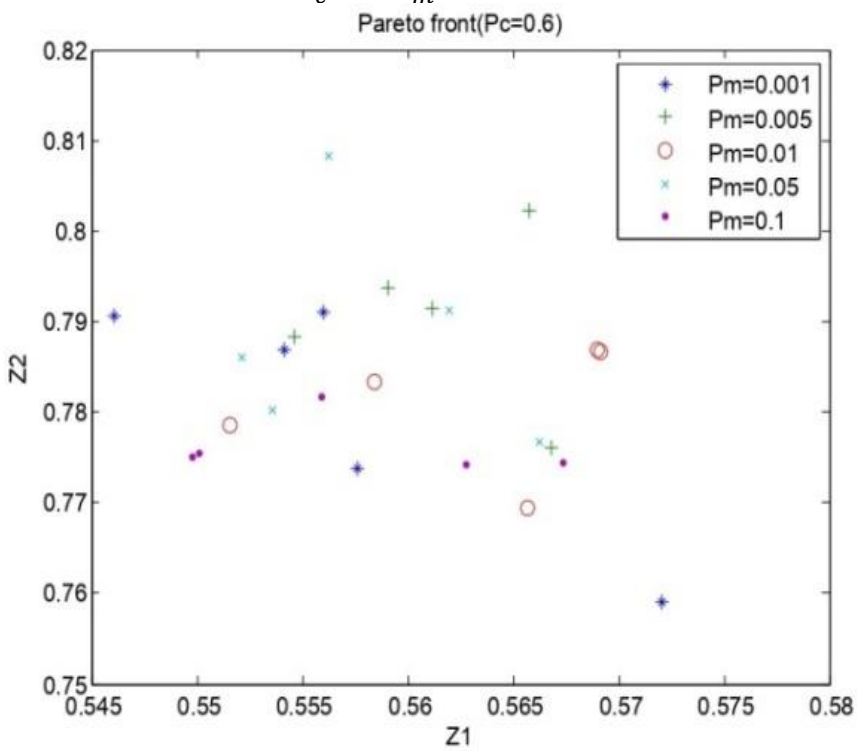

Fig. 2. Pareto Optimal value with $P_{c}=0.6$

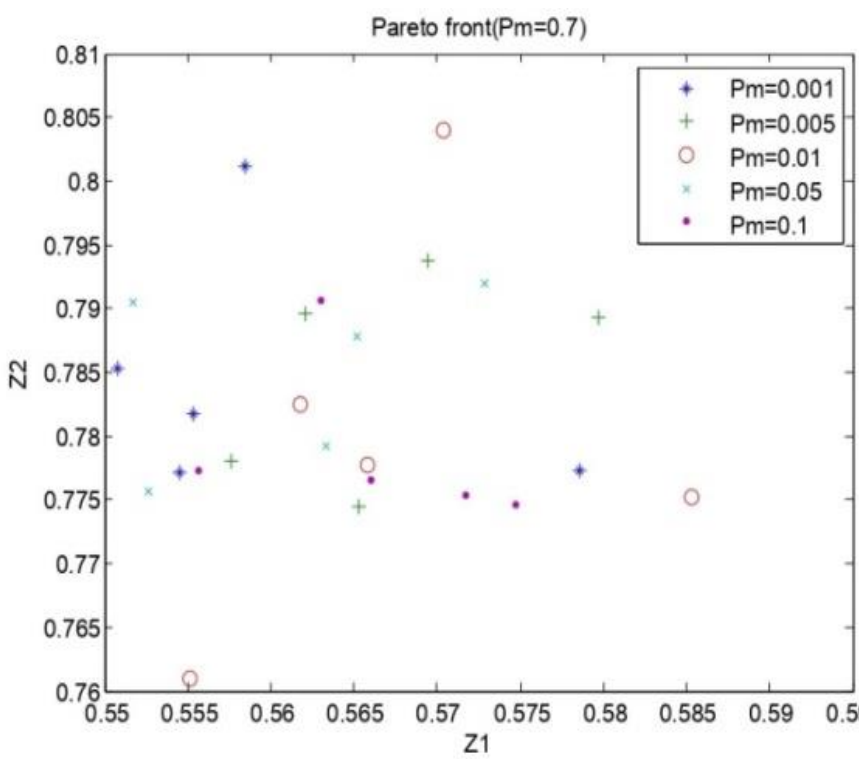

Fig. 3. Pareto Optimal value with $\boldsymbol{P}_{\boldsymbol{c}}=\mathbf{0 . 7}$

Retrieval Number: B3054129219/2019@BEIESP

DOI: 10.35940/ijeat.B3054.129219

Journal Website: www.ijeat.org

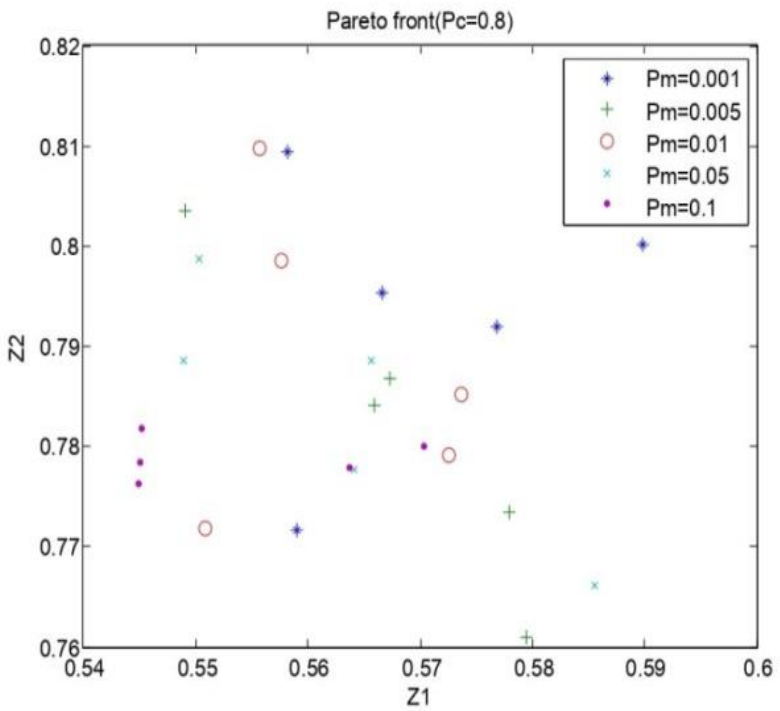

Fig. 4. Pareto Optimal value with $P_{c}=0.8$

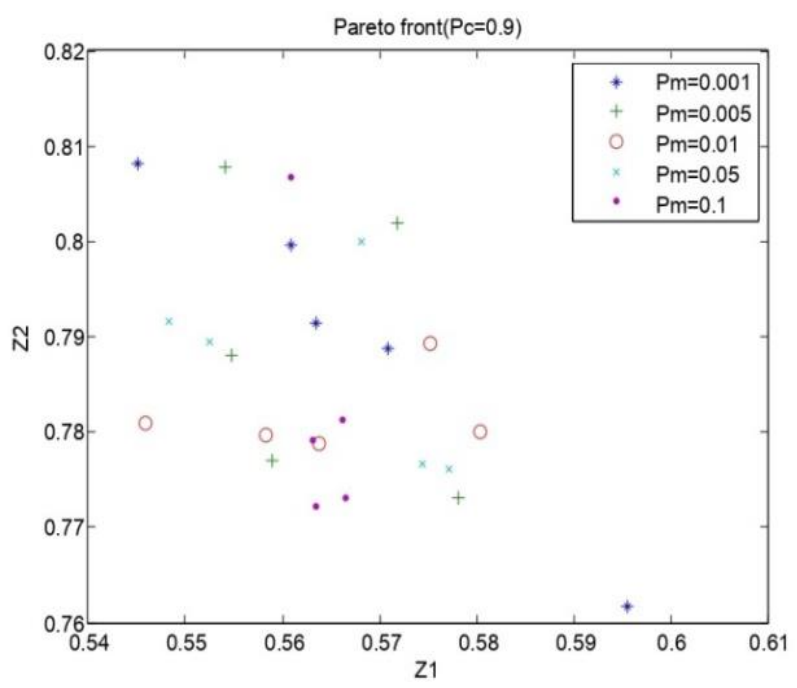

Fig. 5. Pareto Optimal value with $P_{c}=0.9$ 


\begin{tabular}{|c|c|c|c|c|c|c|c|c|c|c|c|c|c|c|c|c|c|c|}
\hline Simulation & $P_{m}$ & $x_{11}$ & $x_{12}$ & $x_{13}$ & $x_{14}$ & $x_{15}$ & $x_{21}$ & $x_{22}$ & $x_{23}$ & $x_{24}$ & $x_{25}$ & $x_{31}$ & $x_{32}$ & $x_{33}$ & $x_{34}$ & $x_{35}$ & $Z^{1}$ & $Z^{2}$ \\
\hline $1 \mathrm{st}$ & 0.001 & 0.2786 & 0.2786 & 0.60606 & 0.65494 & 0.52297 & 0.27077 & 0.6608 & 0.77126 & 0.81036 & 0.02835 & 0.79081 & 0.09384 & 0.04203 & 0.51222 & 0.11241 & 0.5576 & 0.77379 \\
\hline 2nd & 0.001 & 0.33333 & 0.80938 & 0.77615 & 0.9306 & 0.22972 & 0.84262 & 0.6432 & 0.75562 & 0.75073 & 0.03519 & 0.95601 & 0.00684 & 0.82209 & 0.67058 & 0.69111 & 0.5560 & 0.79108 \\
\hline 3 rd & 0.001 & 0.26295 & 0.64321 & 0.04888 & 0.88563 & 0.06549 & 0.42815 & 0.9805 & 0.75562 & 0.65103 & 0.03617 & 0.93842 & 0.01271 & 0.42522 & 0.2131 & 0.47703 & 0.5461 & 0.79069 \\
\hline 4th & 0.001 & 0.13978 & 0.49267 & 0.01662 & 0.60997 & 0.38416 & 0.51026 & 0.8397 & 0.84848 & 0.79961 & 0.02639 & 0.36266 & 0.24633 & 0.20528 & 0.79863 & 0.11241 & 0.57206 & 0.75902 \\
\hline 1 st & 0.005 & 0.54545 & 0.50049 & 0.39296 & 0.42913 & 0.64809 & 0.68328 & 0.4702 & 0.61486 & 0.32551 & 0.06158 & 0.93451 & 0.00195 & 0.98045 & 0.74096 & 1.0 & 0.56574 & 0.80218 \\
\hline 2nd & 0.005 & 0.56207 & 0.84262 & 0.96872 & 0.78495 & 0.00098 & 0.6696 & 0.9062 & 0.12023 & 0.79472 & 0.01075 & 0.60899 & 0.05865 & 0.83089 & 0.84262 & 0.12023 & 0.56682 & 0.77608 \\
\hline 3rd & 0.005 & 0.90225 & 0.71261 & 0.86021 & 0.52981 & 0.12903 & 0.23363 & 0.3314 & 0.27175 & 0.97947 & 0.12512 & 0.95797 & 0.00195 & 0.15933 & 0.63636 & 0.21799 & 0.55465 & 0.78834 \\
\hline 4th & 0.005 & 0.83187 & 0.30303 & 0.5523 & 0.23949 & 0.47312 & 0.59433 & 0.7937 & 0.08798 & 0.71163 & 0.16325 & 0.99413 & 0.01759 & 0.5347 & 0.60313 & 0.59433 & 0.55906 & 0.79365 \\
\hline 1st & 0.01 & 0.11144 & 0.52884 & 0.01564 & 0.2825 & 0.61779 & 0.82014 & 0.9580 & 0.81818 & 0.52297 & 0.15834 & 0.53763 & 0.00489 & 0.21896 & 0.75269 & 0.45943 & 0.5657 & 0.7694 \\
\hline 2nd & 0.01 & 0.16325 & 0.02346 & 0.07625 & 0.69013 & 0.33333 & 0.92375 & 0.6501 & 0.21603 & 0.17498 & 0.35386 & 0.98338 & 0.00391 & 0.53079 & 0.61290 & 0.34311 & 0.56913 & 0.78661 \\
\hline 4th & 0.01 & 0.71359 & 0.95503 & 0.75367 & 0.32063 & 0.8045 & 0.54839 & 0.84457 & 0.89834 & 0.62072 & 0.02151 & 0.33431 & 0.2043 & 0.07722 & 0.12317 & 0.31085 & 0.55157 & 0.77857 \\
\hline $1 \mathrm{st}$ & 0.05 & 0.03324 & 0.05279 & 0.92082 & 0.18671 & 0.83675 & 0.02737 & 0.64712 & 0.16227 & 0.77713 & 0.20723 & 0.57576 & 0.02835 & 0.43891 & 0.13685 & 0.39394 & 0.55213 & 0.78596 \\
\hline 2nd & 0.05 & 0.95406 & 0.19062 & 0.23851 & 0.33822 & 0.0782 & 0.54448 & 0.09873 & 0.79863 & 0.85337 & 0.00195 & 0.95503 & 0.01759 & 0.42913 & 0.82111 & 0.45748 & 0.56194 & 0.79122 \\
\hline 3rd & 0.05 & 0.22385 & 0.43988 & 0.33529 & 0.68622 & 0.90811 & 0.82014 & 0.48876 & 0.10166 & 0.47312 & 0.07136 & 0.66862 & 0.00391 & 0.90518 & 0.04203 & 0.67253 & 0.55627 & 0.80833 \\
\hline 4th & 0.05 & 0.84262 & 0.94135 & 0.52981 & 0.00195 & 0.25122 & 0.94526 & 0.53079 & 0.03519 & 0.98827 & 0.01662 & 0.6393 & 0.06843 & 0.73216 & 0.39198 & 0.02933 & 0.55355 & 0.78025 \\
\hline 1st & 0.1 & 0.02151 & 0.20235 & 0.67742 & 0.14956 & 0.12317 & 0.79277 & 0.4741 & 0.44184 & 0.80254 & 0.00293 & 0.59531 & 0.24438 & 0.52004 & 0.5347 & 0.35875 & 0.55009 & 0.77544 \\
\hline 2nd & 0.1 & 0.478 & 0.4868 & 0.95406 & 0.3001 & 0.70577 & 0.51026 & 0.99609 & 0.06354 & 0.31476 & 0.00587 & 0.75562 & 0.00489 & 0.04594 & 0.70283 & 0.06158 & 0.56739 & 0.77443 \\
\hline $3^{\text {rd }}$ & 0.1 & 0.38514 & 0.348 & 0.97361 & 0.48485 & 0.89052 & 0.75171 & 0.69795 & 0.59726 & 0.64614 & 0.13099 & 0.52884 & 0.02444 & 0.71261 & 0.23754 & 0.00489 & 0.56275 & 0.77425 \\
\hline 4th & 0.1 & 0.09189 & 0.80645 & 0.77615 & 0.16715 & 0.14663 & 0.41251 & 0.11241 & 0.23265 & 0.53763 & 0.03324 & 0.08993 & 0.00684 & 0.56403 & 0.10362 & 0.01466 & 0.54977 & 0.77503 \\
\hline
\end{tabular}

\section{Table VIII: Pareto optimal solutions for $\boldsymbol{P}_{c}=\mathbf{0 . 6}$}

\begin{tabular}{|c|c|c|c|c|c|c|c|c|c|c|c|c|c|c|c|c|c|}
\hline Simulation & $P_{m}$ & $x_{11}$ & $x_{12}$ & $x_{13}$ & $x_{15}$ & $x_{21}$ & $x_{22}$ & $x_{23}$ & $x_{24}$ & $x_{25}$ & $x_{31}$ & $x_{32}$ & $x_{33}$ & $x_{34}$ & $x_{35}$ & $Z^{I}$ & $Z^{2}$ \\
\hline $1 \mathrm{st}$ & 0.001 & 0.4956 & 0.08113 & 0.85435 & 0.89736 & 0.42424 & 0.17595 & 0.79081 & 0.31378 & 0.12415 & 0.7175 & 0.02346 & 0.79179 & 0.05376 & 0.44184 & 0.56083 & 0.79967 \\
\hline 2nd & 0.001 & 0.75758 & 0.07038 & 0.92375 & 0.80156 & 0.44477 & 0.68035 & 0.62561 & 0.07527 & 0.30401 & 0.11437 & 0.02151 & 0.33333 & 0.87879 & 0.01564 & 0.59561 & 0.76178 \\
\hline $3^{\text {rd }}$ & 0.001 & 0.8739 & 0.83773 & 0.69795 & 0.12903 & 0.39687 & 0.17107 & 0.14467 & 0.67351 & 0.10753 & 0.89443 & 0.04399 & 0.29912 & 0.00098 & 0.23558 & 0.5451 & 0.80809 \\
\hline 4th & 0.001 & 0.54839 & 0.66276 & 0.32747 & 0.18084 & 0.27761 & 0.72923 & 0.12512 & 0.3216 & 0.14858 & 0.20723 & 0.0391 & 0.79961 & 0.25415 & 0.53275 & 0.56342 & 0.79144 \\
\hline $1 \mathrm{st}$ & 0.005 & 0.55327 & 0.26197 & 0.62268 & 0.78006 & 0.42033 & 0.55132 & 0.14858 & 0.07429 & 0.18866 & 0.913 & 0.03226 & 0.12219 & 0.22092 & 0.652 & 0.55414 & 0.80777 \\
\hline 2nd & 0.005 & 0.54839 & 0.25122 & 0.28446 & 0.65494 & 0.64516 & 0.99218 & 0.17498 & 0.23949 & 0 & 0.34213 & 0.02346 & 0.14858 & 0.6129 & 0.19648 & 0.5781 & 0.77301 \\
\hline 3rd & 0.005 & 0.91789 & 0.34213 & 0.7957 & 0.34897 & 0.86901 & 0.28055 & 0.63441 & 0.81623 & 0.00391 & 0.3998 & 0.02835 & 0.32258 & 0.37732 & 0.03226 & 0.5589 & 0.77698 \\
\hline 4th & 0.005 & 0.89345 & 0.66178 & 0.17595 & 0.58162 & 0.90616 & 0.26979 & 0.83285 & 0.7781 & 0.02737 & 0.77224 & 0.32845 & 0.54937 & 0.2131 & 0.18768 & 0.55481 & 0.78807 \\
\hline $1 \mathrm{st}$ & 0.01 & 0.26393 & 0.60997 & 0.24927 & 0.23558 & 0.86119 & 0.4565 & 0.07625 & 0.32356 & 0.05376 & 0.5523 & 0.00489 & 0.37243 & 0.94721 & 0.11339 & 0.58041 & 0.77998 \\
\hline 2nd & 0.01 & 0.16813 & 0.88661 & 0.68328 & 0.89541 & 0.87977 & 0.91984 & 0.15543 & 0.61779 & 0.38319 & 0.8436 & 0.02737 & 0.04008 & 0.24731 & 0.36168 & 0.54593 & 0.7808 \\
\hline 3rd & 0.01 & 0.76637 & 0.36266 & 0.80352 & 0.17595 & 0.04008 & 0.69208 & 0.99023 & 0.81036 & 0.03421 & 0.56207 & 933 & 0.03323 & 0.92082 & 0.6002 & 0.5638 & 0.7788 \\
\hline 4th & 0.01 & 0.70381 & 0.57771 & 0.64809 & 0.15249 & 0.739 & 0.56403 & 0.90518 & 0.72336 & 0.01271 & 0.53666 & 0.00782 & 0.77517 & 0.38319 & 0.23069 & 0.55829 & 0.7797 \\
\hline $1 \mathrm{st}$ & 0.05 & 0.2219 & 0.94037 & 0.77126 & 0.03226 & 0.85826 & 0.20723 & 0.71359 & 0.19844 & 0.00098 & 0.26295 & 0.10753 & 0.95601 & 0.81916 & 0.20821 & 0.57445 & 0.77665 \\
\hline 2nd & 0.05 & 0.52981 & 0.59042 & $\begin{array}{l}0.8348 \\
\end{array}$ & 0.00098 & 0.6999 & 0.34702 & 0.37537 & 0.56207 & 0.00195 & 0.28641 & 0.11144 & 0.39003 & 0.59433 & 0.72141 & 0.55253 & 0.78943 \\
\hline 3rd & 0.05 & 0.83382 & 0.30499 & 0.14272 & 0.26295 & 0.3998 & 0.4565 & 0.97165 & 0.72532 & 0.13881 & 0.04008 & 0.00098 & 0.49365 & 0.68231 & 0.46921 & 0.57713 & 0.77613 \\
\hline 4th & 0.05 & 0.10753 & 0.82209 & 0.59726 & 0.57771 & 0.23656 & 0.75073 & 0.93744 & 0.96188 & 0.01369 & 0.44086 & 0.00489 & 0.97752 & 0.04985 & 0.75758 & 0.54832 & $0.7916 \mathrm{~s}$ \\
\hline $1 \mathrm{st}$ & 0.1 & 0.26491 & 0.27468 & 0.49365 & 0.56305 & 0.78397 & 0.39003 & 0.61975 & 0.01662 & 0.0303 & 0.88759 & 0.03519 & 0.81525 & 0.5044 & 0.97263 & 0.5609 & 0.80671 \\
\hline 2nd & 0.1 & 0.11535 & 0.18084 & 0.52102 & 0.17693 & 0.54057 & 0.20919 & 0.68622 & 0.4174 & 0.0088 & 0.14272 & 0.04594 & 0.13587 & 0.50342 & 0.41251 & 0.56308 & 0.77921 \\
\hline 3rd & 0.1 & 0.26784 & 0.21896 & 0.82502 & 0.81036 & 0.93451 & 0.77322 & 0.99805 & 0.11633 & 0.07527 & 0.27859 & 0.00977 & 0.29912 & 0.38416 & 0.31378 & 0.56655 & $0.7730 \mathrm{~s}$ \\
\hline 4th & 0.1 & 0.05865 & 0.78006 & 0.87097 & 0.56305 & 0.76735 & 0.29521 & 0.41153 & 0.52981 & 0.00293 & 0.60704 & 0.02151 & 0.79961 & 0.56501 & 0.06549 & 0.56621 & 0.78128 \\
\hline
\end{tabular}

Table IX: Pareto optimal solutions for $P_{c}=0.7$

Retrieval Number: B3054129219/2019@BEIESP DOI: 10.35940/ijeat.B3054.129219

Journal Website: www.ijeat.org

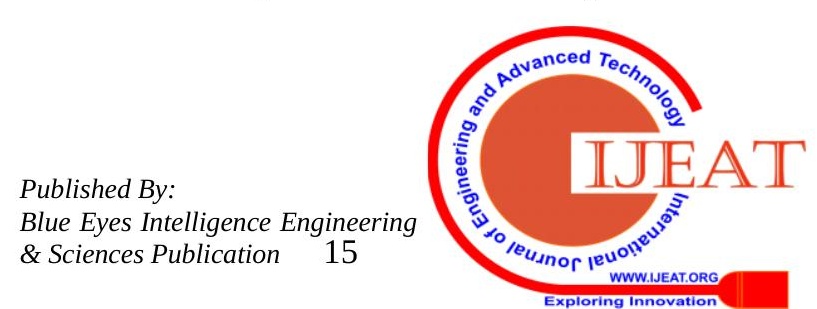


Solving MOLFSTP Involving Normal Distribution Using Simulation Based GA

\begin{tabular}{|c|c|c|c|c|c|c|c|c|c|c|c|c|c|c|c|c|c|}
\hline Simulation & $P_{m}$ & $x_{11}$ & $x_{12}$ & $x_{13}$ & $x_{15}$ & $x_{21}$ & $x_{22}$ & $x_{23}$ & $x_{24}$ & $x_{25}$ & $x_{31}$ & $x_{32}$ & $x_{33}$ & $x_{34}$ & $x_{35}$ & $Z^{I}$ & $Z^{2}$ \\
\hline 1 st & 0.001 & 0.4956 & 0.08113 & 0.85435 & 0.89736 & 0.42424 & 0.17595 & 0.79081 & 0.31378 & 0.12415 & 0.7175 & 0.02346 & 0.79179 & 0.05376 & 0.44184 & 0.56083 & 0.79967 \\
\hline 2nd & 0.001 & 0.75758 & 0.07038 & 0.92375 & 0.80156 & 0.44477 & 0.68035 & 0.62561 & 0.07527 & 0.30401 & 0.11437 & 0.02151 & 0.33333 & 0.87879 & 0.01564 & 0.59561 & 0.76178 \\
\hline $3^{\text {rd }}$ & .001 & 0.8739 & 0.83773 & 0.69795 & 0.12903 & 0.39687 & 0.17107 & 0.14467 & 0.67351 & 0.10753 & 0.89443 & 0.04399 & 0.29912 & 0.00098 & 0.23558 & 0.5451 & 0.80809 \\
\hline 4th & 001 & 0.54839 & 0.66276 & 0.32747 & 0.18084 & 0.27761 & 0.72923 & 0.12512 & 0.3216 & 0.14858 & 0.20723 & 0.0391 & 0.79961 & 0.25415 & 0.53275 & 0.56342 & 0.79144 \\
\hline 1 st & 0.005 & 0.55327 & 0.26197 & 0.62268 & 0.78006 & 0.42033 & 0.55132 & 0.14858 & 0.07429 & 0.18866 & 0.913 & 0.03226 & 0.12219 & 0.22092 & 0.652 & 0.55414 & 0.80777 \\
\hline 2nd & 0.005 & 0.54839 & 0.25122 & 0.28446 & 0.65494 & 0.64516 & 0.99218 & 0.17498 & 0.23949 & 0 & 0.34213 & 0.02346 & 0.14858 & 0.6129 & 0.19648 & 0.5781 & 0.77301 \\
\hline 3rd & 0.005 & 0.91789 & 0.34213 & 0.7957 & 0.34897 & 0.86901 & 0.28055 & 0.63441 & 0.81623 & 0.00391 & 0.3998 & 0.02835 & 0.32258 & 0.37732 & 0.03226 & 0.5589 & 0.77698 \\
\hline 4th & 0.005 & 0.89345 & 0.66178 & 0.17595 & 0.58162 & 0.90616 & 0.26979 & 0.83285 & 0.7781 & 0.02737 & 0.77224 & 0.32845 & 0.54937 & 0.2131 & 0.18768 & 0.55481 & 0.78807 \\
\hline 1 st & 0.01 & 0.26393 & 0.60997 & 0.24927 & 0.23558 & 0.86119 & 0.4565 & 0.07625 & 0.32356 & 0.05376 & 0.5523 & 0.00489 & 0.37243 & 0.94721 & 0.11339 & 0.58041 & 0.77998 \\
\hline 2nd & 0.01 & 0.16813 & 0.88661 & 0.68328 & 0.89541 & 0.87977 & 0.91984 & 0.15543 & 0.61779 & 0.38319 & 0.8436 & 0.02737 & 0.04008 & 0.24731 & 0.36168 & 0.54593 & 0.78084 \\
\hline 3rd & 0.01 & 0.76637 & 0.36266 & 0.80352 & 0.17595 & 0.04008 & 0.69208 & 0.99023 & 0.81036 & 0.03421 & 0.56207 & 0.02933 & 0.03323 & 0.92082 & 0.6002 & 0.5638 & 0.77884 \\
\hline 4th & 0.01 & 0.70381 & 0.57771 & 0.64809 & 0.15249 & 0.739 & 0.56403 & 0.90518 & 0.72336 & 0.01271 & 0.53666 & 0.00782 & 0.77517 & 0.38319 & 0.23069 & 0.55829 & 0.7797 \\
\hline $1 \mathrm{st}$ & 0.05 & 0.2219 & 0.94037 & 0.77126 & 0.03226 & 0.85826 & 0.20723 & 0.71359 & 0.19844 & 0.00098 & 0.26295 & 0.10753 & 0.95601 & 0.81916 & 0.20821 & 0.57445 & 0.77665 \\
\hline 2nd & 0.05 & 0.52981 & 0.59042 & 0.8348 & 0.00098 & 0.6999 & 0.34702 & 0.37537 & 0.56207 & 0.00195 & 0.28641 & 0.11144 & 0.39003 & 0.59433 & 0.72141 & 0.55253 & 0.78943 \\
\hline 3rd & 0.05 & 0.83382 & 0.30499 & 0.14272 & 0.26295 & 0.3998 & 0.4565 & 0.97165 & 0.72532 & 0.13881 & 0.04008 & 0.00098 & 0.49365 & 0.68231 & 0.46921 & 0.57713 & 0.77613 \\
\hline 4th & 0.05 & 0.10753 & 0.82209 & 0.59726 & 0.57771 & 0.23656 & 0.75073 & 0.93744 & 0.96188 & 0.01369 & 0.44086 & 0.00489 & 0.97752 & 0.04985 & 0.75758 & 0.54832 & 0.79169 \\
\hline 1st & 0.1 & 0.26491 & 0.27468 & 0.49365 & 0.56305 & 0.78397 & 0.39003 & 0.61975 & 0.01662 & 0.0303 & 0.88759 & 0.03519 & 0.81525 & 0.5044 & 0.97263 & 0.5609 & 0.80671 \\
\hline 2nd & 0.1 & 0.11535 & 0.18084 & 0.52102 & 0.17693 & 0.54057 & 0.20919 & 0.68622 & 0.4174 & 0.0088 & 0.14272 & 0.04594 & 0.13587 & 0.50342 & 0.41251 & 0.56308 & 0.77921 \\
\hline 3rd & 0.1 & 0.26784 & 0.21896 & 0.82502 & 0.81036 & 0.93451 & 0.77322 & 0.99805 & 0.11633 & 0.07527 & 0.27859 & 0.00977 & 0.29912 & 0.38416 & 0.31378 & 0.56655 & 0.77309 \\
\hline 4th & 0.1 & 0.05865 & 0.78006 & 0.87097 & 0.56305 & 0.76735 & 0.29521 & 0.41153 & 0.52981 & 0.00293 & 0.60704 & 0.02151 & 0.79961 & 0.56501 & 0.06549 & 0.56621 & 0.78128 \\
\hline
\end{tabular}

Table X: Pareto optimal for $P_{c}=0.8$

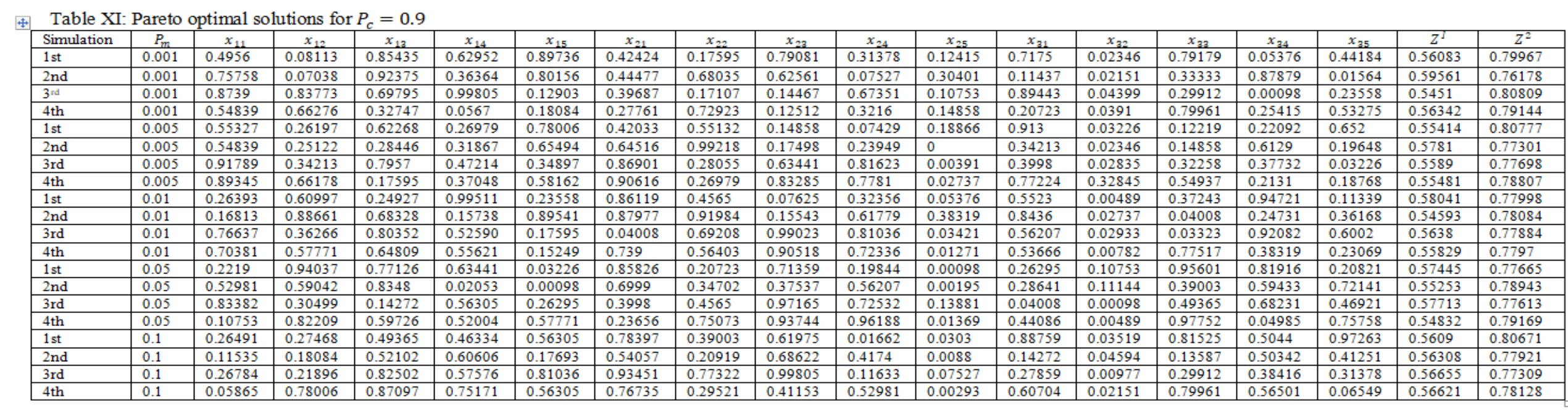

Table XI: Pareto optimal solutions for $\boldsymbol{P}_{c}=\mathbf{0 . 9}$

Retrieval Number: B3054129219/2019@BEIESP DOI: 10.35940/ijeat.B3054.129219

Journal Website: www.ijeat.org
Published By:

Blue Eyes Intelligence Engineering

\& Sciences Publication 16

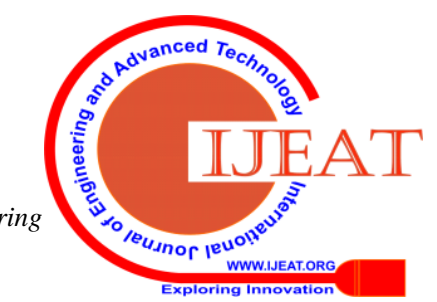




\section{REFERENCE}

1. A. Trivedi, D. Srinivasan, K. Sanyal and A. Ghosh, "A survey of multiobjective evolutionary algorithms based on decomposition," IEEE Transactions on Evolutionary Computation, vol. 21, no. 3, 2017, pp. 440-462.

2. K. Swarup, "Transportation technique in linear fractional functional programming," Journal of royal naval scientific service, vol. 21, no. 5 , 1966, pp. 256-260.

3. R.S. Porchelvi and A.A. Sheela, "A new algorithmic approach to linear multi-objective fractional transportation problem," International Journal of Scientific and Engineering Research, vol. 6, no. 3, 2015, pp. 229-231.

4. S. Sadia, N. Gupta and Q. M. Ali, "Multiobjective capacitated fractional transportation problem with mixed constraints," Mathematical Sciences Letters, vol. 5, no. 3, 2016, pp.235-242.

5. M. Safi and M.G. Seyyed, "Uncertainty in linear fractional transportation problem," International Journal of Nonlinear Analysis and Applications, vol. 8, no. 1, 2017, pp. 81-93.

6. A. Khurana and S.R. Arora, "The sum of a linear and a linear fractional transportation problem with restricted and enhanced flow," Journal of Interdisciplinary Mathematics, vol. 9, no. 2, 2006, pp. 373-383.

7. M. Sivri, I. Emiroglu, C. Guler and F. Tasci, "A solution proposal to the transportation problem with the linear fractional objective function," (In Modeling, Simulation and Applied Optimization (ICMSAO), 2011 4th International Conference; IEEE; 2011), 2011, pp. 1-9.

8. K. Gupta and S.R. Arora, "An algorithm to find optimum cost time trade of pairs in a fractional capacitated transportation problem with restricted flow," International Journal Of Research In Social Sciences, vol. 2, no. 2, 2012, pp. 418.

9. N. G uzel, Y. Emiroglu, F. Tapci, C. Guler and M. Syvry, “A solution proposal to the interval fractional transportation problem," Applied Mathematics \& Information Sciences, vol. 6, no. 3, 2012, pp. 567-571.

10. A. Pradhan and M.P. Biswal, "Computational methodology for linear fractional transportation problem," (Proceedings of the 2015 Winter Simulation Conference; IEEE Press; 2015), 2015, pp. 3158-3159.

11. B. Liu, "Uncertainty theory," Uncertainty theory, 2007, pp. 205-234.

12. H. Guo, X. Wang and S. Zhou, "A transportation problem with uncertain costs and random supplies," International Journal of e-Navigation and Maritime Economy, vol. 2, 2015, pp. 1-11.

13. Y. Sheng and Y. Kai, "A transportation model with uncertain costs and demands," Information: An international interdisciplinary journal, vol. 15, no. 8, 2012, pp. 3179-3186.

14. G.B. Dantzig, "Linear programming under uncertainty," Management Science, vol. 1, no. 3 \& 4, 1955, pp. 197-206.

15. A. Goicoechea and D. Lucien, "Nonnormal deterministic equivalents and a transformation in stochastic mathematical programming," Applied mathematics and computation, vol. 21, no. 1, 1987, pp 51-72.

16. R. Jagannathan, "Chance-constrained programming with joint constraints," Operations Research, vol. 22, no. 2, 1974, pp. 358-372.

17. V. Charles and D. Dutta, "Linear Stochastic Fractional Programming with Sum of Probabilistic Fractional Objective," Optimization Online, 2005.

18. V. Charles and D. Dutta, “Extremization of multi-objective stochastic fractional programming problem," Annals of Operations Research, vol. 143, no. 1, 2006, pp. 297-304.

19. S. Jain and A. Nitin, "An inverse transportation problem with the linear fractional objective function," Advanced Modelling \& Optimization, vol. 15, no. 3, 2013, pp.677-687.

20. V.A. Jadhav and D.M. Doke, "Solution Procedure to Solve Fractional Transportation Problem with Fuzzy Cost and Profit Coefficients," International Journal of Mathematics, vol. 4, no. 7, 2016.

21. S. Javaid, S.A. Jalil, and Z. Asim, "A model for uncertain multi-objective transportation problem with fractional objectives," Int J Oper Res., vol. 14, no. 1, 2017, pp. 11-25.

22. J. Holland, Adaptation in natural and artificial systems: an introductory analysis with application to biology, Control and artificial intelligence. University of Michigan Press, 1975.

23. G.A. Vignaux and Z. Michalewicz, "A genetic algorithm for the linear transportation problem," IEEE transactions on systems, man, and cybernetics, vol. 21, no. 2, 1991, pp. 445-452.

24. A. Syarif and M. Gen, "Genetic algorithm for nonlinear side constrained transportation problem," (Proceedings of International Conference on Computers; 2000), 2000.

25. K. Bharathi, and C. Vijayalakshmi, "Optimization of Multi-objective Transportation Problem Using Evolutionary Algorithms," Global Journal of Pure and Applied Mathematics, vol. 12, no. 2, 2016, pp. 1387-1396.
26. D. Dutta, S. Acharya and M. Rajashree, "Genetic algorithm based fuzzy stochastic transportation programming problem with continuous random variables," Opsearch, vol. 53, no. 4, 2016, pp. 835-872.

27. E. Ardjmand, et al. "Applying genetic algorithm to a new bi-objective stochastic model for transportation, location, and allocation of hazardous materials," Expert systems with applications, vol. 51, 2016, pp. 49-58.

28. T. Karthy and K. Ganesan, "Multi-objective transportation problem Genetic Algorithm approach," International Journal of Pure and Applied Mathematics, vol. 119, no. 9, 2018, pp. 343-350.

\section{AUTHORS PROFILE}

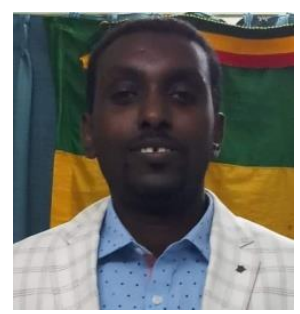

Adane Abebaw Gessesse received his BSc and MSc degree in Applied Mathematics from University of Gondar and Addis Ababa University, Ethiopia in 2010 and 2012 respectively. He is a research scholar in the Department of Mathematics, School of Applied Sciences at KIIT, deemed to University, Bhubaneswar, India. Before joining, deemed to University for Ph.D. program, he was served as a lecturer in Debre Tabor University, Debre Tabor, Ethiopia. He has authored or coauthored more than four research papers in journals and conferences. He has presented his research works in national and international conferences. His current areas of research interests are linear programming, nonlinear programming, mixed integer programming, multi-objective programming, fuzzy programming, genetic algorithm, stochastic programming and transportation problem.

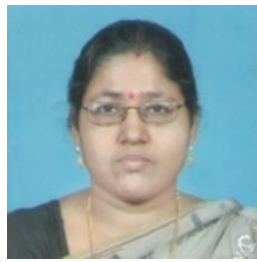

Dr. Rajashree Mishra She is currently working as Assistant Professor in Department of Mathematics, School of Applied Sciences, KIIT University, Bhubaneswar, Odisha. Prior to that she was working as Assistant Professor in Mathematics in VITAM College of Engineering, Vishakhapatnam, Andhrapradesh. She received her Ph. D Degree from KIIT University in 2014 and completed M. Sc Degree in Mathematics in the year 1999 from Utkal University, Bhubaneswar. Her areas of research interest are Evolutionary Computing which specifically includes (Genetic Algorithm and Bacterial Foraging Optimization), Fuzzy probabilistic Programming and Multi-objective non linear optimization. She is having publications in reputed journals. She has presented research papers and participated in many National and International Conference of repute. She is also the Reviewer to many International Conferences. She has also co-guided one research scholar and 3 are ongoing.

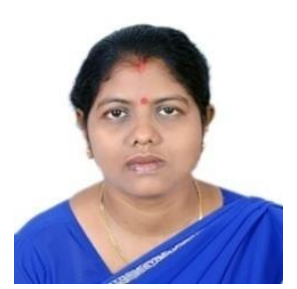

Dr. Mitali Madhumita Acharya (Sahani) is an Assistant Professor in the Department of Mathematics, KIIT University, Bhubaneswar, India. She was born in 1980 in Odisha. She has received her $\mathrm{PhD}$ degree in Numerical Functional Analysis from Indian Institute of Technology Kharagpur, India in the year 2011. Her PhD thesis was on "Spectral methods for integral equations". Before Joining as a Research Scholar in IIT Kharagpur for PhD program, she has received her MPhil and MSc degrees in Mathematics from Berhampur University and Ravenshaw (Autonomous) College in the year 2005 and 2003, respectively. She has published a good number of research papers in International journals in Numerical Functional Analysis. Presently she is also working in Operations Research as well as Numerical Functional Analysis. Her research areas are operator theory, integral equations, approximation theory, numerical functional analysis, optimization techniques etc. 